\title{
Orbital transfers in an asteroid system considering the solar radiation pressure
}

\author{
Geraldo Magela Couto Oliveira ${ }^{1,2}$ - Antonio F. Bertachini de A. Prado ${ }^{2}$. \\ Diogo Merguizo Sanchez ${ }^{2}$ Vivian Martins Gomes ${ }^{3}$
}

Received: 30 March 2017 / Accepted: 17 August 2017 / Published online: 7 September 2017

(C) Springer Science+Business Media B.V. 2017

\begin{abstract}
The present paper studies the effects of the radiation pressure in the trajectories of a spacecraft in transfers between the collinear Lagrange points of a double asteroid system. The system considered is this paper is formed by the double asteroid $1996 \mathrm{FG}_{3}$ and the maneuvers are always assumed to be bi-impulsive. In a system formed by asteroids, the solar radiation pressure has a significant influence in the transfers paths. This occurs because the gravitational forces in these systems are smaller if compared with systems formed by larger bodies. Solutions with lower and higher fuel consumption can be found by adding the solar radiation pressure. The radiation pressure was not used as a control but its effects over the transfers were measured. For a small system of primaries such as an asteroid system, it is very important to take into account this force to make sure that the spacecraft will reach the desired point.
\end{abstract}

Keywords Astrodynamics · Double asteroids · Lagrange points $\cdot$ Solar radiation pressure $\cdot$ Orbital transfers

\section{Introduction}

In recent years, the interest in asteroids and comets has increased and several missions have been proposed for these bodies, such as NEAR Shoemaker (Prockter et al. 2002), Dawn (Rayman et al. 2006), Hayabusa (Kawaguchi

G.M.C. Oliveira

magela@cefetmg.br

1 Federal Center for Technological Education of Minas Gerais (CEFET-MG), Contagem, Brazil

2 National Institute for Space Research (INPE), São José dos Campos, Brazil

3 São Paulo State University (FEG/UNESP), Guaratinguetá, Brazil et al. 2006), Rosetta (Glassmeier et al. 2007), Aster mission (Sukhanov et al. 2010), Hayabusa 2 (Tsuda et al. 2013b) and MarcoPolo-R (Michel et al. 2014). Because those bodies have high eccentricities, they have periapsis near the Sun and apoapsis more distant, so the influence of the solar radiation pressure on the probes of these missions may become relevant. In addition, with the wide range of asteroid-Sun distances covered during an orbital period, the dynamics become more complex.

In two of the missions mentioned above, Aster and MarcoPolo-R, the proposed target is a system of asteroids, which allows these missions to study various bodies in a single mission. In order to extend the duration of the mission and to reduce the fuel consumed, it is important to study the behavior of the probe during the orbital maneuvers in the asteroids systems.

Previous works considered the problem of orbital maneuvers between the equilibrium Lagrange points of the Sun-Earth system (Broucke 1979), the Earth-Moon system (Prado 1996; Oliveira et al. 2016) and Sun-Earth-Moon system (Cabette and Prado 2008). In a recent work (Yang et al. 2015), the problem of orbit transfers connecting equilibrium points of irregular-shaped asteroids were studied. It was considered only the gravity of the asteroid in their dynamics. The perturbations from the gravity of the Sun and the planets were considered very small compared with the mass-point gravitational forces of the asteroids. It was not considered the case when the asteroid is very close to a planet.

This work will study the same problem of orbit transfers between the equilibrium Lagrange points mentioned before. The system considered here is a double asteroid. Beyond the effects of the gravity that were used in previous publications, in this work the effects of the solar radiation pressure on such transfers is taken into account. The objective is to demonstrate that the solar radiation pressure must be con- 
sidered when performing maneuvers under the conditions mentioned above, otherwise the spacecraft will not reach the desired points.

It is also suggested that, by changing the area/mass ratio of the spacecraft and the distance to the Sun, it is possible to observe the importance and the influence of this effect over the trajectories. The present paper shows the results for simulations of orbital transfers between the collinear Lagrange points of the double asteroid $1996 \mathrm{FG}_{3}$ (Wolters et al. 2011; Scheirich et al. 2015), which is the target of the MarcoPolo$\mathrm{R}$ mission.

\section{Mathematical model}

In this work, the planar circular restricted three-body problem is combined with the forces coming from the solar radiation pressure to find different orbital trajectories necessary to move the spacecraft between the collinear Lagrange points of the asteroid system $1996 \mathrm{FG}_{3}$. The primaries $\mathrm{M}_{1}$ (the main asteroid) and $\mathrm{M}_{2}$ (the smaller asteroid) orbit the common center of mass of the system in a circular Keplerian orbit, and the third body $\mathrm{M}_{3}$ (the spacecraft), considered to have a negligible mass, orbits the main bodies. The motion of $\mathrm{M}_{3}$ does not affect the motion of the main bodies $\mathrm{M}_{1}$ and $\mathbf{M}_{2}$, but it is affected by them (Szebehely 1967).

The equations of motion can be written in a system of non-dimensional units, also known as a canonical system of units. In this system:

1. The gravitational constant $(\mathrm{G})$ is one.

2. The unit of distance is assumed as the distance between the two primaries (the semi-major axis of their orbits).

3 . The unit of time considered, $2 \pi$, is defined as the period of the motion of the two primaries.

4. The mass of the primaries are given by the mass ratio. The primary $\mathrm{M}_{2}$ has a given mass of $\mu=\mathrm{M}_{2} /\left(\mathrm{M}_{1}+\mathrm{M}_{2}\right)$, and the primary $\mathrm{M}_{1}$ has a given mass of $(1-\mu)$. It makes the total mass one.

5. The angular velocity of the motion of the primaries is considered one.

Table 1 shows the values for these parameters for the system considered in this paper, which is the binary asteroid $1996 \mathrm{FG}_{3}$, where $\mathrm{M}_{1}$ is the main asteroid (Alpha) and $\mathrm{M}_{2}$ is the smaller one (Beta).

Table 1 Canonical system of units for the binary asteroid $1996 \mathrm{FG}_{3}$ (Wolters et al. 2011; Scheirich et al. 2015)

\begin{tabular}{ll}
\hline Unit of distance & $2.8 \mathrm{~km}$ \\
Unit of time & $6.00 \times 10^{4} \mathrm{~s}$ \\
Unit of velocity & $2.93 \times 10^{-4} \mathrm{~km} / \mathrm{s}$ \\
\hline
\end{tabular}

The equations of motion of $\mathrm{M}_{3}$, which is represented in the rotating coordinate system, are given by Szebehely (1967):

$\ddot{x}-2 \dot{y}=\frac{\partial \Omega}{\partial x}+a_{\text {srp }, x}$,
$\ddot{y}+2 \dot{x}=\frac{\partial \Omega}{\partial y}+a_{\text {srp }, y}$,

where $\Omega$ is the pseudo-potential given by

$\Omega=\frac{1}{2}\left(x^{2}+y^{2}\right)+\frac{1-\mu}{r_{1}}+\frac{\mu}{r_{2}}$,

where

$r_{1}^{2}=(x+\mu)^{2}+y^{2}$,

$r_{2}^{2}=(x-1+\mu)^{2}+y^{2}$,

and $a_{s r p, x}$ and $a_{s r p, y}$ are the components of the solar radiation pressure that is now added as a perturbation. The magnitude of the acceleration due to the solar radiation pressure is given by Vallado (2001):

$\vec{a}_{s r p}=-C_{r} \frac{P_{s}}{c} \frac{A_{s}}{m r^{2}} \hat{r}$

where:

- the negative sign indicates that the radiation force points to the opposite direction of the Sun;

- $C_{r}$ is the reflectivity coefficient of the surface, which may vary from 0 to 2 ;

- $P_{S}$ is the solar constant, equals to $1353 \mathrm{~W} / \mathrm{m}^{2}$ at one A.U. (Astronomical Unit, which is the Sun-Earth distance);

- $c$ is the speed of light;

- $A_{s}$ is the area of the spacecraft illuminated by the Sun;

- $m$ is the mass of the spacecraft;

- $r$ is the distance Sun-spacecraft;

- the unit vector $\hat{r}$ represents the direction of the acceleration, which is assumed to be the Sun-spacecraft line.

Numerical integration is used to solve this problem because the (1a) and (1b) have no analytical solutions. The solution of this problem is obtained using the TPBVP (Two Point Boundary Value Problem) which is solved using gradient methods and numerical integrations (Press et al. 1989).

To solve the TPBVP, in this work, the following steps are used as in Prado (1996):

1. The initial state is given by the initial velocity $\vec{v}_{i}$ and the initial prescribed position $\vec{r}_{i}$.

2. The equations of motion are integrated from the initial time $\tau_{0}=0$ until the final time $\tau_{f}$.

3. The final position $\vec{r}_{f}$ is checked. It is calculated from the numerical integration with the final time and the prescribed final position with the time of flight. If there is 
a difference less than a specified tolerance, the solution is found and this process stops. Otherwise the process returns to step 1 and an increment in the initial guessed velocity $\vec{v}_{i}$ and in the guessed final time are made.

The standard gradient method is used to find the increment in the guessed variables, as it is in Press et al. (1989). Minor modifications were made in the routines available in this reference.

\section{Conditions for the proposed transfers}

The maneuvers are treated as bi-impulsive, and starts with the application of the first impulse at the initial position of the spacecraft, the initial Lagrange point, and ends with the application of the second impulse in the final position of the spacecraft, the final Lagrange point.

Figure 1 shows how the transfers are simulated. Considering that the spacecraft is positioned on a Langrange point of the double asteroid system $\left(\mathrm{M}_{1}\right.$ and $\left.\mathrm{M}_{2}\right)$, it will be required that the spacecraft changes its orbit during the mission, such that it may study and collect information about the bodies of this system. It may be also possible that the spacecraft can collect solar material when the mission approaches the closest point of its orbit around the Sun, at the perihelion.

An example for a transfer from the Lagrange point $\mathrm{L}_{1}$ to Lagrangre point $\mathrm{L}_{3}$ is shown in Fig. 1. In this example, the spacecraft is situated at point $\mathrm{L}_{1}$ and must reach point $\mathrm{L}_{3}$. In this way, an impulsive maneuver is applied at point $\mathrm{L}_{1}$, giving the spacecraft the necessary variation of velocity $\Delta v_{1}$ to start the transfer orbit 1 , directed to point $\mathrm{L}_{3}$. When the spacecraft reaches point $\mathrm{L}_{3}$, another impulsive maneuver is applied, giving the spacecraft the necessary variation of velocity $\Delta v_{1}{ }^{\prime}$, in order to put the spacecraft in the desired final orbit, the Lagrange point $\mathrm{L}_{3}$. In our scenario, it is assumed that the spacecraft needs to return to $\mathrm{L}_{1}$. Thus, by applying a variation of velocity $\Delta v$ at point $\mathrm{L}_{3}$, and a variation of velocity $\Delta v_{2}{ }^{\prime}$, at the end point $\mathrm{L}_{1}$, the spacecraft will realize the transfer orbit 2, returning to the Lagrange point $\mathrm{L}_{1}$.

Another key point that is considered in the simulations made here is the initial position of the Sun at the beginning of the maneuvers. In order to measure and verify the effects of the solar radiation pressure, it is simulated five different cases. In the first case the solar radiation pressure is not considered. The next cases consider the Sun in four different positions with respect to the asteroid system when the maneuvers are followed. It is assumed that the Sun is situated at 0 degree, 90 degrees, 180 degrees and 270 degrees with respect to the asteroid system. These locations are shown in Fig. 2.

In Fig. 2, $\mathrm{M}_{1}$ represents the larger asteroid (Alpha), $\mathrm{M}_{2}$ the smaller one (Beta) and $\mathrm{M}_{3}$ the spacecraft. The five Lagrange points of this system are also represented.

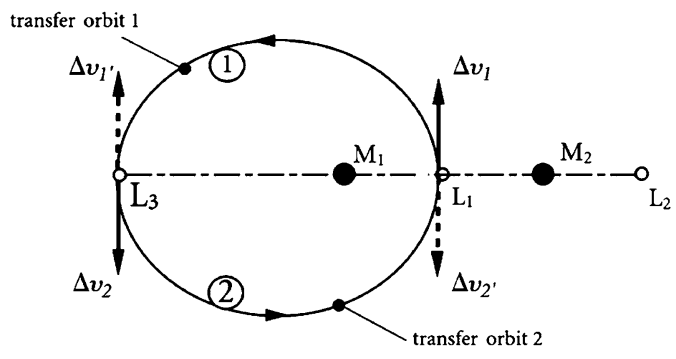

Fig. 1 Bi-impulsive transfers between the Lagrange points $\mathrm{L}_{1}$ and $\mathrm{L}_{3}$

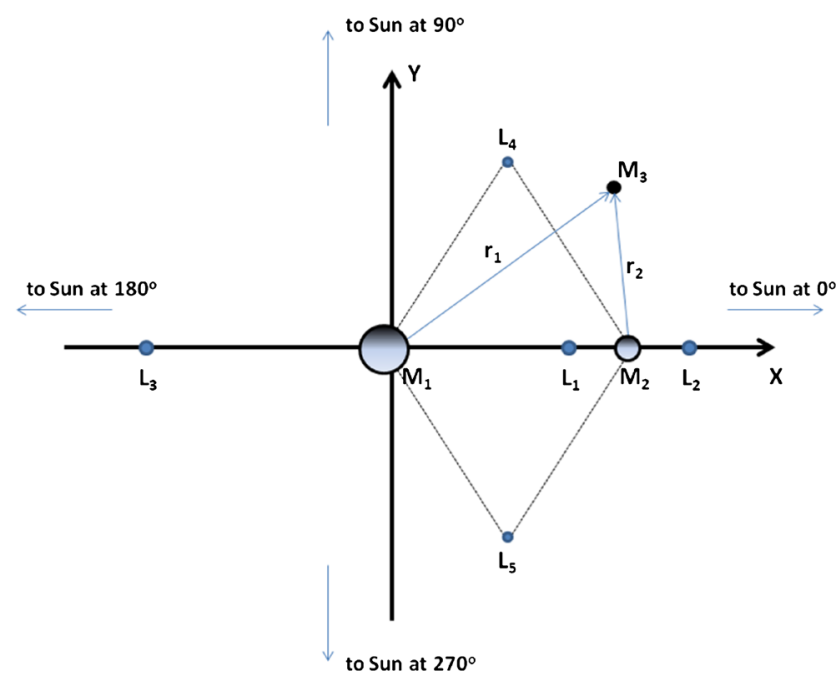

Fig. 2 Location of the Sun with respect to the primaries

The next constraint considered in the simulations is the area/mass ratio. Three values of area/mass are used: $0.02 \mathrm{~m}^{2} / \mathrm{kg}, 0.1 \mathrm{~m}^{2} / \mathrm{kg}$ and $0.5 \mathrm{~m}^{2} / \mathrm{kg}$. This wide range of values are considered to verify the effects of the solar radiation pressure over many types of missions. A mission with a simple spacecraft and no or small solar panels has a small area/mass ratio, but when it is considered a mission with a larger panel, for example to study and catch particles from the solar wind, the area/mass ratio increases.

The Japanese mission Hayabusa (Kawaguchi et al. 2006), from the Japanese Space Agency (JAXA), was launched in 2003 to collect a surface sample of material from the small asteroid Itokawa (1998 SF36) and returned with samples to the Earth for analysis in 2010. This spacecraft had a boxshaped main body and two solar panel wings with a total array area of $12 \mathrm{~m}^{2}$. The launched mass was $510 \mathrm{~kg}$, which gives an area/mass ratio of $0.023 \mathrm{~m}^{2} / \mathrm{kg}$. A new mission, Hayabusa 2 (Tsuda et al. 2013b), was designed to rendezvous with asteroid Ryugu (1999 JU3) and return a sample. It was launched in 2014 and the sample will be returned to the Earth in late 2020. This mission is similar in design to the first Hayabusa mission, it has the same $12 \mathrm{~m}^{2}$ solar panel wings, however it is $90 \mathrm{~kg}$ heavier and has increased redundancy and more scientific instruments for proximity obser- 
vation of $1999 \mathrm{JU} 3$. The launch mass was $600 \mathrm{~kg}$, which gives an area/mass ratio of $0.02 \mathrm{~m}^{2} / \mathrm{kg}$.

In addition to the two Hayabusa missions, the Japanese space agency has sent, in 2010, the IKAROS (Tsuda et al. 2013a) probe to Venus and tested, for the first time, the concept of solar sail in an interplanetary mission. The solar sail of IKAROS was a huge square which sides of 14 meters and 20 meters in a diagonal line, after deployed. Therefore, with a panel measuring 14 meters $\times 14$ meters, totaling $196 \mathrm{~m}^{2}$ of area, and a mass of approximately $310 \mathrm{~kg}$, it had an area/mass ratio of approximately $0.63 \mathrm{~m}^{2} / \mathrm{kg}$.

One approach for a mission that would go closer to the Sun is that it could carry a solar sail and collect material from the Sun and, when it returns to the Earth, this material could be recovered and studied. The Genesis mission (Lo et al. 1998) was launched in 2001 and returned to Earth in 2004 with material collected from the solar wind. The Genesis spacecraft reached a Halo orbit around the Sun-Earth Lagrange point $\mathrm{L}_{1}$, which is about 0.989 A.U. from the Sun. The asteroid system $1996 \mathrm{FG}_{3}$ has an elliptical orbit, which has its periapsis at 0.685 A.U. from the Sun. In this way, a spacecraft that has this asteroid system as a target will reach a distance much closer to the Sun than the distance reached by the Genesis mission. Thus, such mission could study the asteroid system and also collect material from the solar wind more efficiently.

The asteroid $1996 \mathrm{FG}_{3}$ has an elliptical orbit, so, in order to verify the influence of the solar radiation pressure on the spacecraft during the transfers between both asteroids, two points of the orbit of the asteroid are considered for the simulations, the closest and the furthest approach to the Sun. The periapsis (0.685 A.U.) and the apoapsis (1.423 A.U.), were chosen to verify how influent the solar radiation pressure is in its maximum and minimum values.

\section{Results}

As mentioned above, the purpose of this work is to study orbital transfers around the binary asteroid $1996 \mathrm{FG}_{3}$. Thus, some simulations of orbital transfers between the collinear Lagrange points of this system have been done. As a result, it is shown the plots with the variation of velocity $\Delta v$ against time and the variation of velocity $\Delta v$ against the initial flight path angle (fpa), as done in Prado (1996). The definition of this angle is such that the zero is in the " $\mathrm{X}$ " axis pointing to the positive direction and it increases in the counterclockwise sense. This definition is shown Fig. 3.

It is also presented, in Tables 4 to 9 , the values that indicate the minimums found for $\Delta v$, the fpa and the respective transfer times for the four different initial position of the Sun. It is also included one more case, which is when the solar radiation pressure is neglected. This is done to clearly show the effects of the solar radiation pressure.

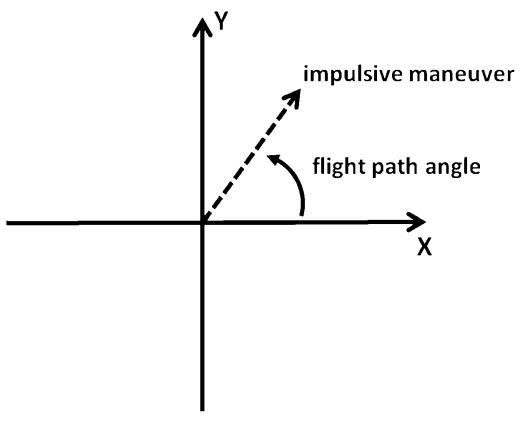

Fig. 3 Flight path angle $(f p a)$.

The results are organized in figures and tables. Figures present the time and initial flight path angle required to perform the maneuver and its correspondent variation of velocity $\Delta v$, as well as some of the trajectories. The variation of velocity required by the maneuvers gives a number more directly related to the fuel consumption, but it requires hypothesis related to the velocity of the spacecraft at the end points of the transfer in the initial and the final orbits. On the other hand, the tables show the minimum $\Delta v$, the $f p a$ and the correspondent times for each transfer considered at periapsis. The plots of the $\Delta v$ against time and $\Delta v$ against initial flight path angle are in the rotating frame. The values of area/mass considered vary from $0.02 \mathrm{~m}^{2} / \mathrm{kg}$ to $0.5 \mathrm{~m}^{2} / \mathrm{kg}$.

Figure 4 shows the required $\Delta v$ to move the probe from the Lagrange point $\mathrm{L}_{1}$ to $\mathrm{L}_{3}$, when the asteroid is at its periapsis and the solar radiation pressure is not considered. Figure $4 \mathrm{a}$ plots the $\Delta v$ as a function of time and Fig. $4 \mathrm{~b}$ plots the $\Delta v$ as a function of the fpa. Figure $4 \mathrm{c}$ shows the trajectories for the minimum and maximum variation of velocity. Note that the trajectory for the minimum $\Delta v$ is direct, passing very close to Alpha what requires low values of $\Delta v_{1}$ and $\Delta v_{2}$ resulting in a lower final $\Delta v$. However, the trajectory for the maximum $\Delta v$ makes a complete revolution before reaching $\mathrm{L}_{3}$ what requires high values of $\Delta v_{1}$ and $\Delta v_{2}$ resulting in a higher final $\Delta v$.

To verify the influence of the solar radiation pressure over the transfers it is used the maneuver with the minimum $\Delta v$, the flight path angle of 174.113 degrees and the time transfer of 0.258 days. Four cases of the initial position of the Sun with respect to the asteroid are considered.

Figure 5 shows the trajectories from $\mathrm{L}_{1}$ to $\mathrm{L}_{3}$ for the three cases of area/mass: $0.02,0.1$ and $0.5 \mathrm{~m}^{2} / \mathrm{kg}$. It is possible to verify that the spacecraft does not reach the final Lagrange point $\mathrm{L}_{3}$, due to the solar radiation pressure. The final position reached by the probe depends of the initial position of the Sun. This figure clearly shows the expected effects of the solar radiation pressure. When the maneuver is calculated based in a dynamics without solar radiation pressure, Lagrange point $\mathrm{L}_{3}$ is reached exactly, based in the numerical accuracy defined. In this case, the acceleration of the spacecraft is $1.05 \times 10^{-12} \mathrm{~m} / \mathrm{s}^{2}$. When in- 


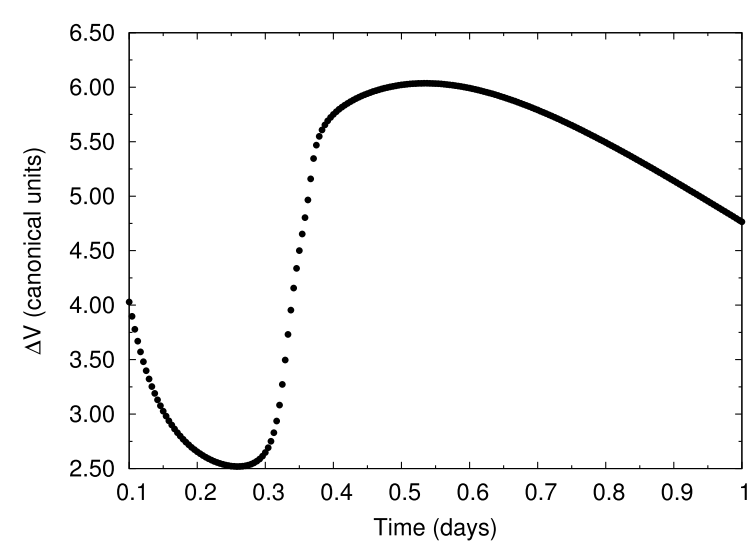

(a) $\Delta v$ as function of time.

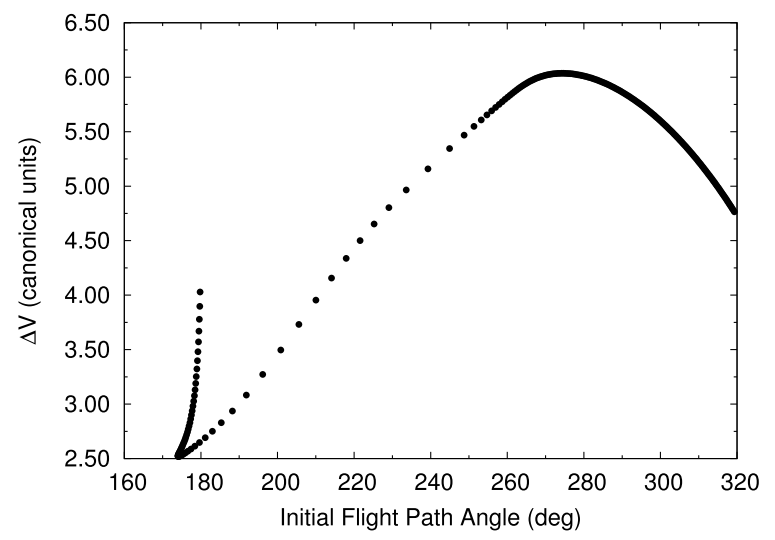

(b) $\Delta v$ as function of the flight path angle.

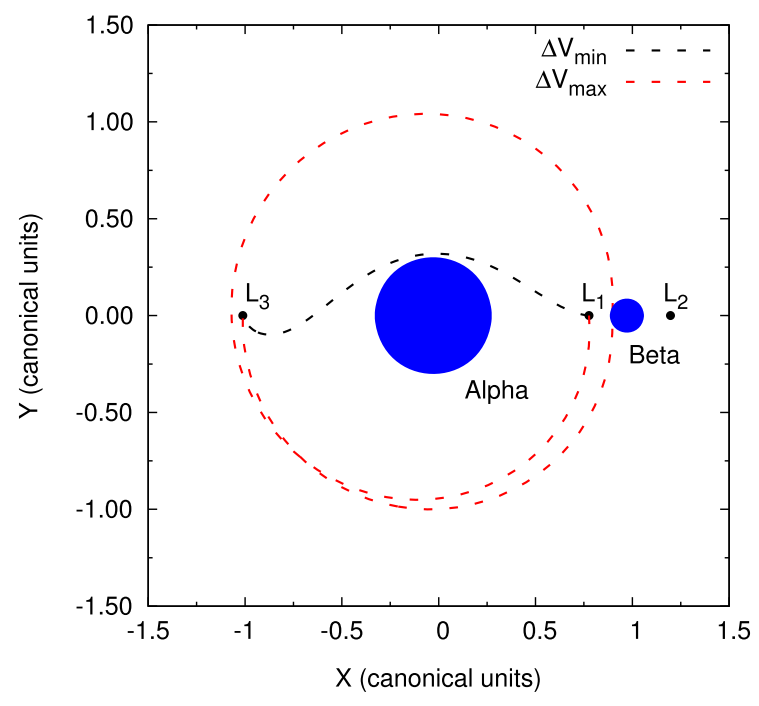

(c) Trajectories from $\mathrm{L}_{1}$ to $\mathrm{L}_{3}$ for $\Delta v_{\min }$ and $\Delta v_{\max }$.

Fig. 4 Transfers from $L_{1}$ to $L_{3}$ with the asteroid at periapsis and excluding the solar radiation pressure

cluding the solar radiation pressure with the Sun at 0 degrees there is an extra force in the direction of the motion of the probe. The probe is accelerated and it passes by the Lagrange point $\mathrm{L}_{3}$. For an area/mass of $0.02 \mathrm{~m}^{2} / \mathrm{kg}$ this acceleration is $2.25 \times 10^{-5} \mathrm{~m} / \mathrm{s}^{2}$, for an area/mass of $0.1 \mathrm{~m}^{2} / \mathrm{kg}$ this acceleration is $2.33 \times 10^{-5} \mathrm{~m} / \mathrm{s}^{2}$ and for an area/mass of $0.5 \mathrm{~m}^{2} / \mathrm{kg}$ this acceleration is $2.75 \times 10^{-5} \mathrm{~m} / \mathrm{s}^{2}$. These new values of acceleration are greater than the one without solar radiation pressure. Of course the magnitude of deviation depends on the area/mass ratio. For larger values the deviation is larger, as shown in the sequence a, b, c of Fig. 5 . When the Sun is at 90 degrees, there is an extra force pointing to the negative direction of the vertical axis. The probe is shifted to the bottom of the plot, also the negative direction of the vertical axis, proportional to the area/mass ratio. It is noted that this shift is large enough to cause a collision between the spacecraft and Alpha (Fig. 5c). In the situation where the Sun is at 180 degrees, the solar radiation pressure points in the direction opposite to the motion of the probe, so it is decelerated and does not reach the Lagrange point $\mathrm{L}_{3}$. These deviations are proportional to the area/mass ratio. When the Sun is at 270 degrees, there is an extra force pointing to the positive direction of the vertical axis and the probe is shifted to the top of the plot, also the positive direction of the vertical axis. The shifts are also proportional to the area/mass ratio. It is also noted the occurrence of a collision with Alpha. Therefore, Fig. 5 shows very well the importance of considering the solar radiation pressure in the dynamical model.

Table 2 shows the minimum distances from $\mathrm{L}_{3}$ reached by the probe according to the initial position of the Sun. Table 2 shows in detail the effects of the solar radiation pressure in the error at the final point of the maneuver. If the probe has an area/mass $=0.02 \mathrm{~m}^{2} / \mathrm{kg}$, the minimum distance between the probe and the Lagrange point goes from 26.79 meters to 132.72 meters, depending on 


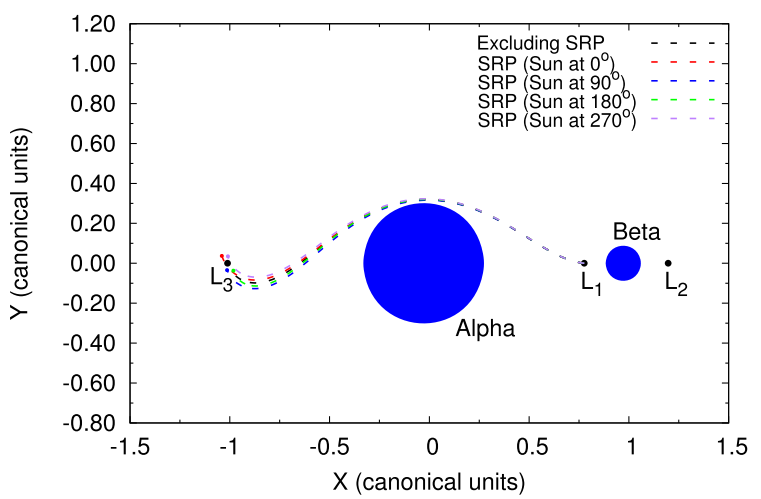

(a) $\mathrm{L}_{1}$ to $\mathrm{L}_{3}, \mathrm{~A} / \mathrm{m}=0.02 \mathrm{~m}^{2} / \mathrm{kg}$

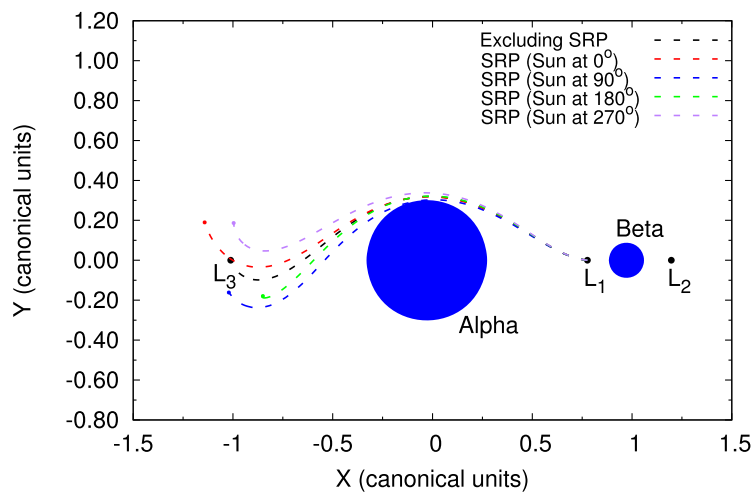

(b) $\mathrm{L}_{1}$ to $\mathrm{L}_{3}, \mathrm{~A} / \mathrm{m}=0.1 \mathrm{~m}^{2} / \mathrm{kg}$

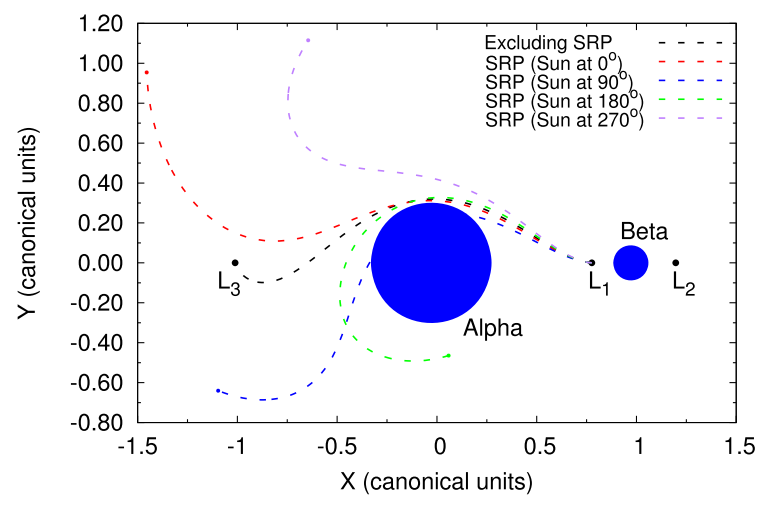

(c) $\mathrm{L}_{1}$ to $\mathrm{L}_{3}, \mathrm{~A} / \mathrm{m}=0.5 \mathrm{~m}^{2} / \mathrm{kg}$

Fig. 5 Trajectories from $L_{1}$ to $L_{3}$ with the asteroid at periapsis of its orbit

Table 2 Minimum distances from $\mathrm{L}_{3}$

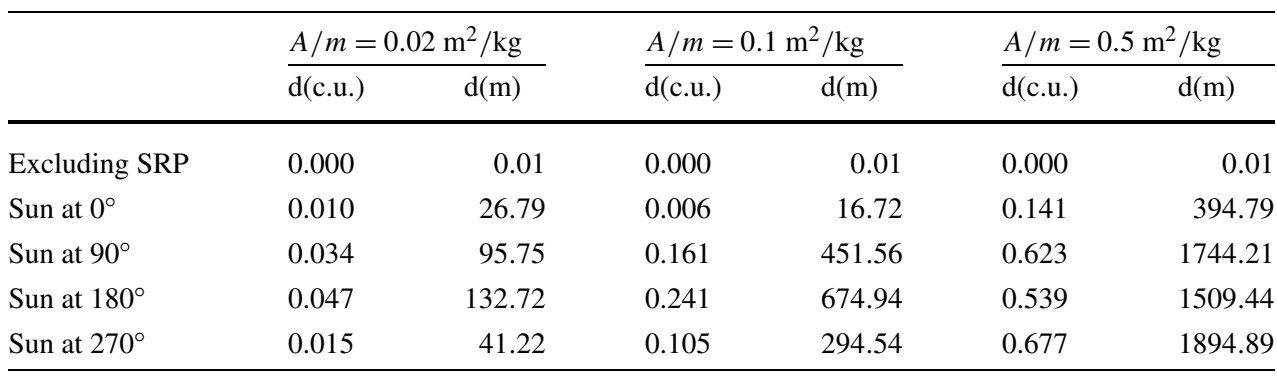

the initial position of the Sun. As expected, the minimum error occurs when the Sun is initially at 0 degrees, with the probe being accelerated. The maximum error occurs when the Sun is initially at 180 degrees, with the probe being decelerated and the effect on the deviation of the trajectory is very large. Those values increases very much with the area/mass ratio, reaching levels of 674.94 meters for an area/mass $=0.1 \mathrm{~m}^{2} / \mathrm{kg}$ and 1894.89 meters for an area $/ \mathrm{mass}=0.5 \mathrm{~m}^{2} / \mathrm{kg}$. It is also noted that, for this highest value of the area/mass ratio, the errors are stronger when the Sun is initially at the positions 90 and 270 degrees. The trajectories shown in Fig. 5 identify the reason. With a such large solar radiation pressure, this force deviates too much the trajectories during the transfer, sending the probe away from $\mathrm{L}_{3}$.

The next transfer considers the way back, $\mathrm{L}_{3}$ to $\mathrm{L}_{1}$. Figure 6 shows the required $\Delta v$ to move the probe from the Lagrange point $\mathrm{L}_{3}$ to $\mathrm{L}_{1}$ when the asteroid is at periapsis and the solar radiation pressure is not considered. The minimum $\Delta v$ found is 2.519 canonical units, at the transfer time of 0.258 days. These are the same values found for the $L_{1}$ to $\mathrm{L}_{3}$ transfer, but the flight path angle of 71.815 degrees is different, since the direction of motion is opposite.

Figure 7 shows the plot of the trajectories from $\mathrm{L}_{3}$ to $\mathrm{L}_{1}$ for the three cases of area/mass: $0.02,0.1$ and $0.5 \mathrm{~m}^{2} / \mathrm{kg}$. The $\Delta v_{1}$ considered for these simulations are the minimum ones, and the total time used for the transfers is 0.258 days, 


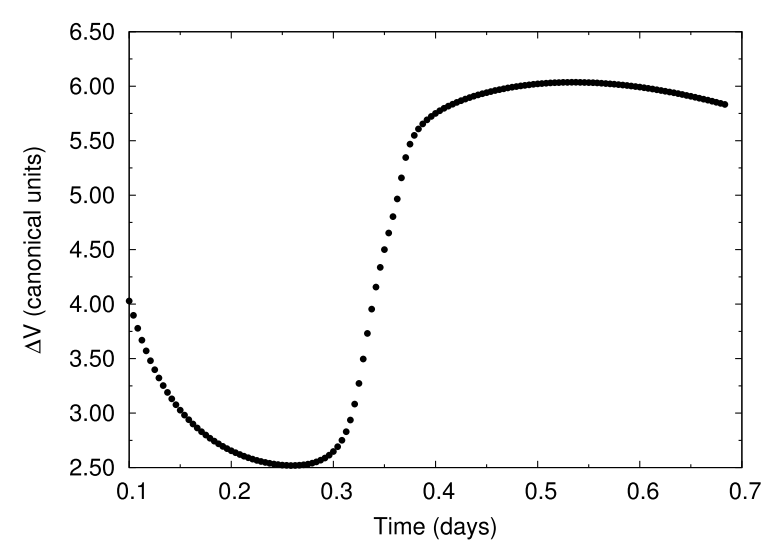

(a) $\Delta v$ as function of time.

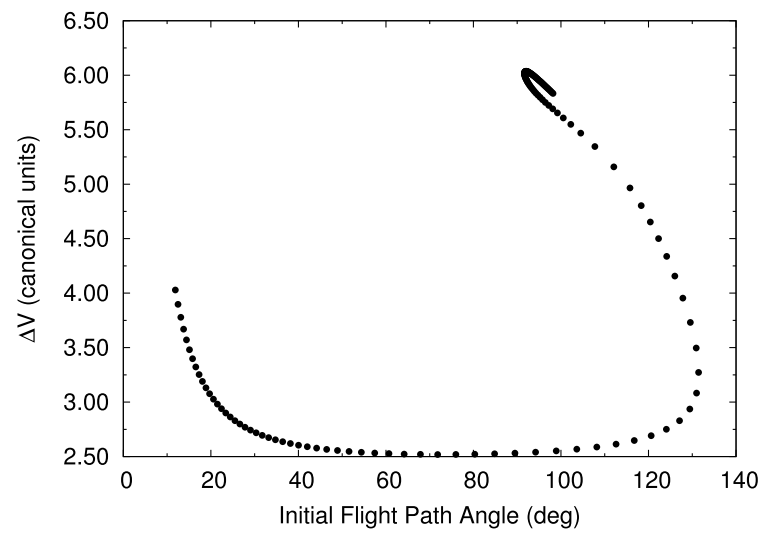

(b) $\Delta v$ as function of the flight path angle.

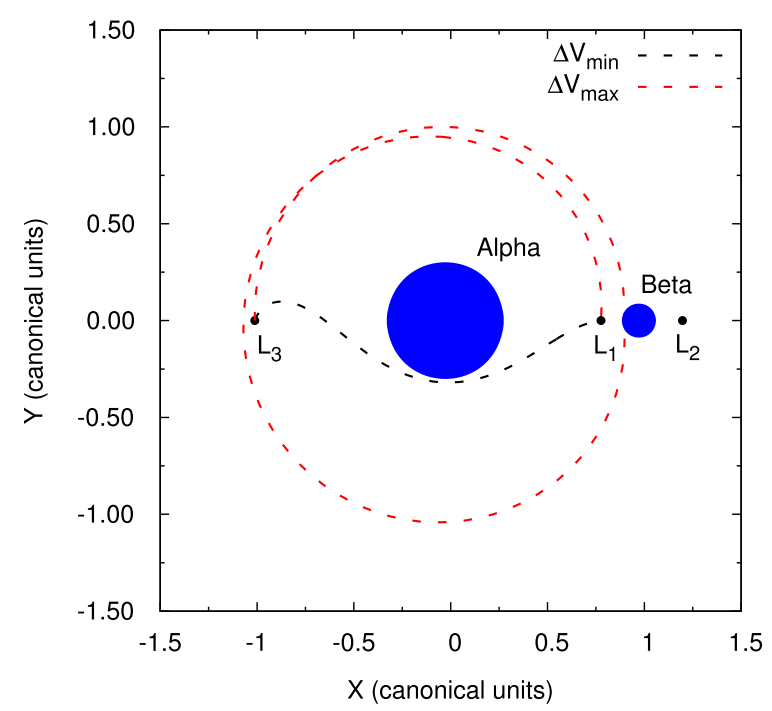

(c) Trajectories from $\mathrm{L}_{3}$ to $\mathrm{L}_{1}$ for $\Delta v_{\min }$ and $\Delta v_{\max }$.

Fig. 6 Transfer from $L_{3}$ to $L_{1}$ with the asteroid at periapsis and excluding the solar radiation pressure

Table 3 Minimum distances from $L_{1}$

\begin{tabular}{|c|c|c|c|c|c|c|}
\hline & \multicolumn{2}{|c|}{$A / m=0.02 \mathrm{~m}^{2} / \mathrm{kg}$} & \multicolumn{2}{|c|}{$A / m=0.1 \mathrm{~m}^{2} / \mathrm{kg}$} & \multicolumn{2}{|c|}{$A / m=0.5 \mathrm{~m}^{2} / \mathrm{kg}$} \\
\hline & d(c.u.) & $\mathrm{d}(\mathrm{m})$ & $\overline{\mathrm{d} \text { (c.u.) }}$ & $\mathrm{d}(\mathrm{m})$ & $\overline{\mathrm{d} \text { (c.u.) }}$ & $\mathrm{d}(\mathrm{m})$ \\
\hline Excluding SRP & 0.000 & 0.01 & 0.000 & 0.01 & 0.000 & 0.01 \\
\hline Sun at $0^{\circ}$ & 0.020 & 55.40 & 0.093 & 261.27 & 0.456 & 1275.53 \\
\hline Sun at $90^{\circ}$ & 0.056 & 158.11 & 0.313 & 872.89 & 1.320 & 3696.92 \\
\hline Sun at $180^{\circ}$ & 0.010 & 28.15 & 0.034 & 94.59 & 0.095 & 265.18 \\
\hline Sun at $270^{\circ}$ & 0.052 & 146.41 & 0.240 & 673.21 & 0.608 & 1701.10 \\
\hline
\end{tabular}

the same for $\mathrm{L}_{1}$ to $\mathrm{L}_{3}$. For all these five cases, the initial flight path angle is the same, 71.815 degrees. Figure 7 has the same physical explanations made for Fig. 5, just the direction of motion is different. The probe now moves from the left to the right of the plots, so the acceleration of the probe occurs when the Sun is at 180 degrees and the deceleration occurs when the Sun is at 0 degree. The same types of shifts and occurrence of collisions with Alpha occur.
As found previously, it is possible to verify that the spacecraft does not reach the final Lagrange point $\mathrm{L}_{1}$, due to the solar radiation pressure. The final position reached by the probe depends on the initial position of the Sun. Table 3 shows the minimum distances from $\mathrm{L}_{1}$ reached by the probe according to the initial position of the Sun. Table 3 has a very similar interpretation of Table 2 . The only difference is the inversion of the minimum errors. The Sun, initially lo- 


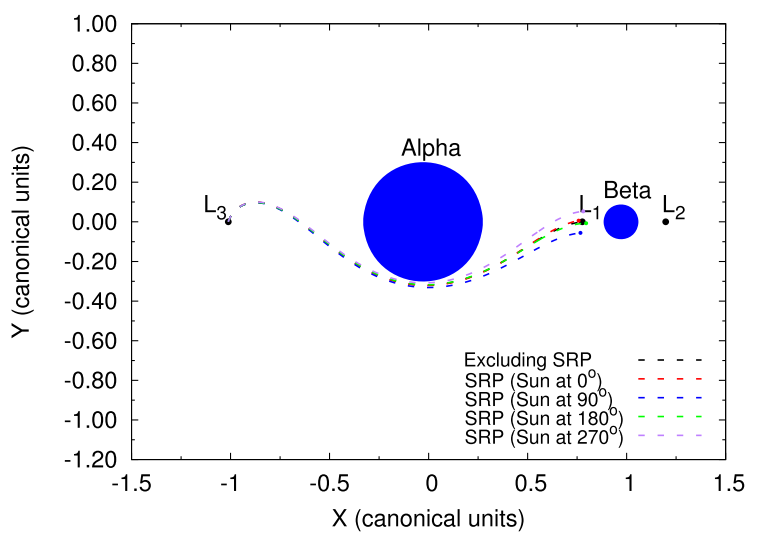

(a) $\mathrm{L}_{3}$ to $\mathrm{L}_{1}, \mathrm{~A} / \mathrm{m}=0.02 \mathrm{~m}^{2} / \mathrm{kg}$

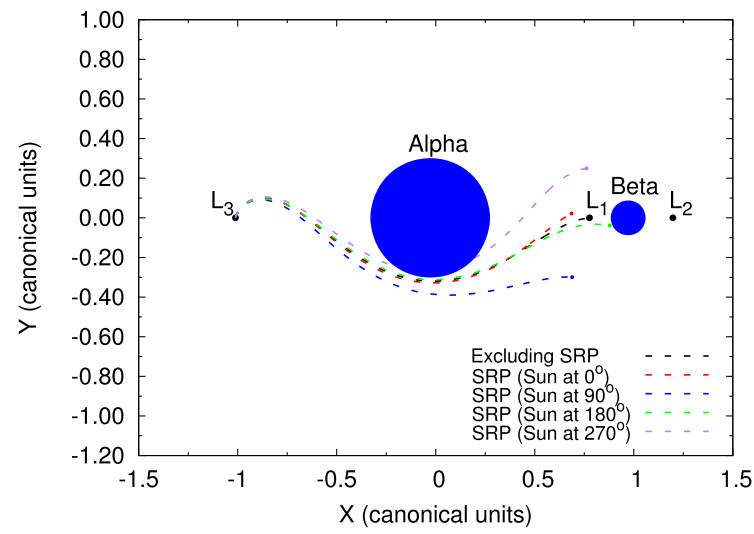

(b) $\mathrm{L}_{3}$ to $\mathrm{L}_{1}, \mathrm{~A} / \mathrm{m}=0.1 \mathrm{~m}^{2} / \mathrm{kg}$

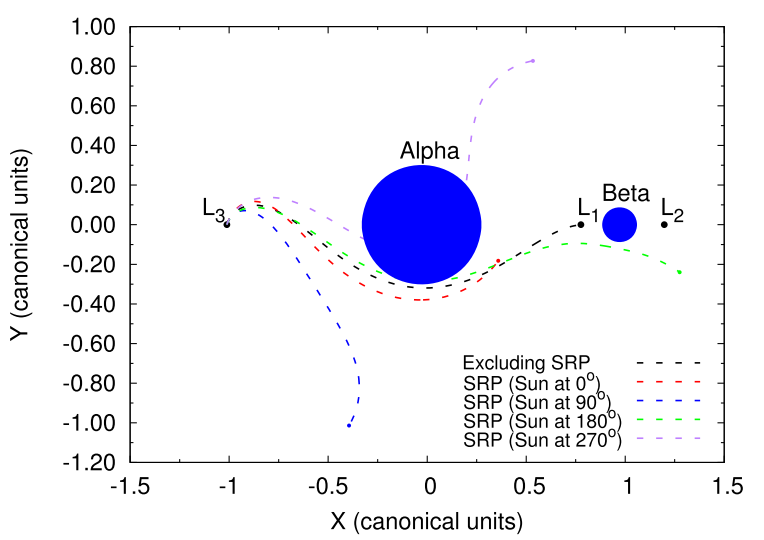

(c) $\mathrm{L}_{3}$ to $\mathrm{L}_{1}, \mathrm{~A} / \mathrm{m}=0.5 \mathrm{~m}^{2} / \mathrm{kg}$

Fig. 7 Trajectories from $L_{3}$ to $L_{1}$ with the asteroid at periapsis of its orbit

cated in 180 degrees, now accelerated the probe and has the minimum errors for the lower values of the area/mass ratio. The maximum errors are located when the Sun is at the initial position of 0 degree, so decelerating the probe. For the highest value of the area/mass, the smallest errors occur when the Sun is at the positions 90 and 270 degrees, as occurred also in Table 2, but there is an inversion, and the case of 90 degrees now has larger errors.

As a conclusion, the solar radiation pressure must be considered in the dynamics for the transfers, otherwise the spacecraft will not reach the desired Lagrange point in the end of the transfer. The next simulations show the real values of $\Delta v$ required to achieve the desired final Lagrange point.

Figures 8 and 9 show in details the behavior of the velocity increment as a function of time and the flight path angle. It is possible to find the best transfers for every value of the area/mass ratio and initial position of the Sun. The discontinuities of the lines indicate points where there is a change of family of transfers. One family ended due to a collision with Alpha and the algorithm then needs to find a different family of transfers, with very different initial parameters.

Figure 8 shows the results for transfers when the asteroid is in the periapsis of its orbit around the Sun. It shows how $\Delta v$ changes due to the area/mass ratio. As could be expected, the higher it turns, more changes in the plots of $\Delta v$ are made.

Table 4 shows a comparison of $\Delta v_{\text {min }}$, fpa and transfer time for all five cases for the initial position of the Sun. Table 4 shows a different view of this problem. It identifies the best maneuver to be made under the solar radiation pressure effects. In other words, it shows how to use the solar radiation pressure in favor of minimizing the fuel consumed required by the maneuver. The first line of this table shows the basic results, which are the ones valid for the situation with no solar radiation pressure. It is shown that the minimum magnitude of the impulse to be applied depends on the initial position of the Sun. Those magnitudes can be smaller than the one valid for the case without the solar radiation pressure, so helping to make the maneuver with less fuel; or higher, making the maneuver to be more expensive. It means that the choice of the time to perform the maneuver, so the initial position of the Sun, makes a difference in the fuel consumption of the maneuver. Those differences are of the order of plus 0.025 in the worst case and minus 0.023 in the best case, for an area/mass ratio of $0.02 \mathrm{~m}^{2}$. Those numbers are much higher for the other values of area/mass ratio, 


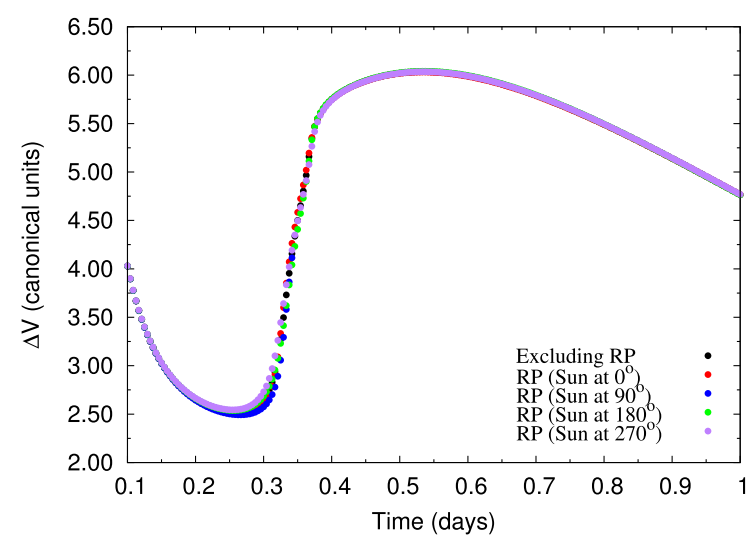

(a) $\Delta v$ as function of time. $\mathrm{A} / \mathrm{m}=0.02 \mathrm{~m}^{2} / \mathrm{kg}$

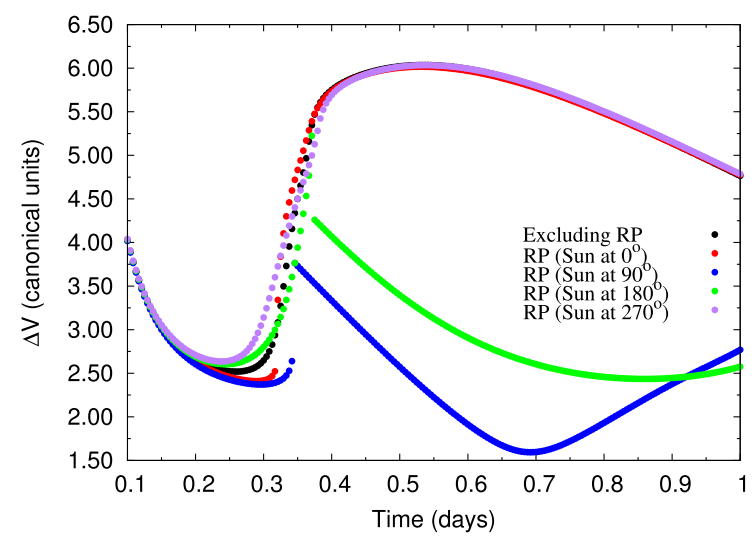

(c) $\Delta v$ as function of time. $\mathrm{A} / \mathrm{m}=0.1 \mathrm{~m}^{2} / \mathrm{kg}$

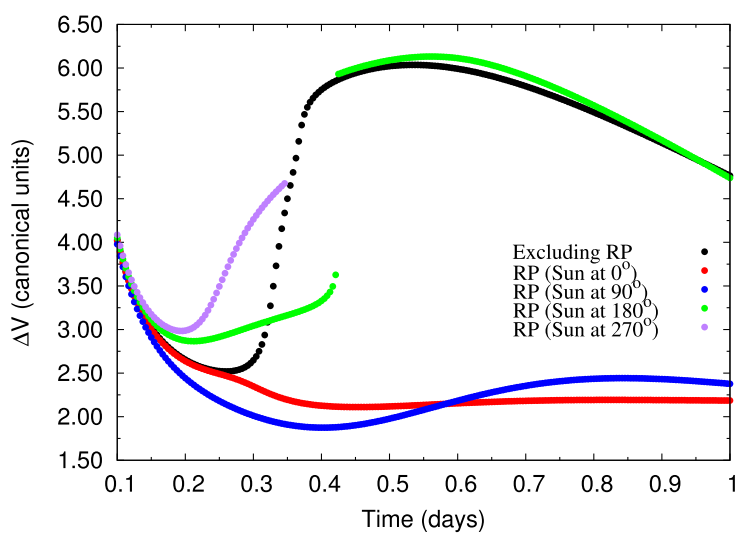

(e) $\Delta v$ as function of time. $\mathrm{A} / \mathrm{m}=0.5 \mathrm{~m}^{2} / \mathrm{kg}$

Fig. 8 Transfers from $L_{1}$ to $L_{3}$ with the asteroid at periapsis of its orbit

reaching savings of 0.926 (about 37\%) for an area/mass ratio of $0.1 \mathrm{~m}^{2}$ and 0.645 (about 26\%) for an area/mass ratio of $0.5 \mathrm{~m}^{2}$.

On the other side, if a wrong choice is made for the time to start the transfer, the extra variations of velocity required are of the order of 0.116 (about $5 \%$ ) for an area/mass ratio of $0.1 \mathrm{~m}^{2}$ and 0.464 (about 18\%) for an area/mass ratio of $0.5 \mathrm{~m}^{2}$. It means differences between the best and the

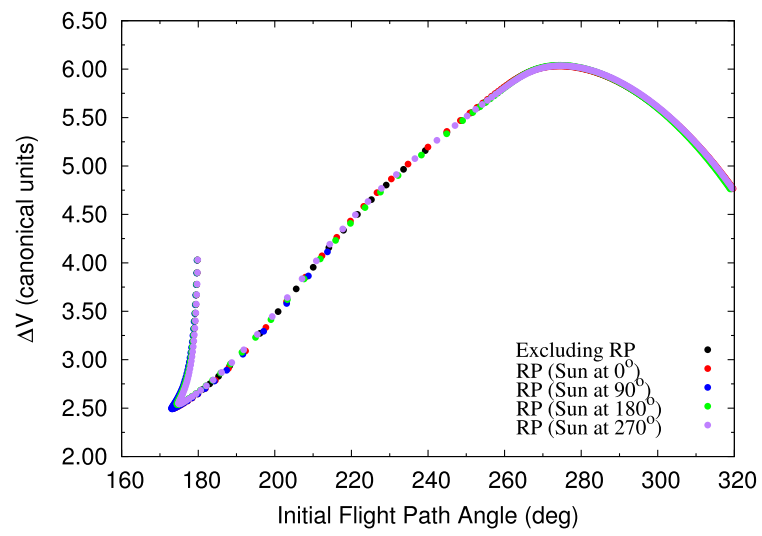

(b) $\Delta v$ as function of $f p a . \mathrm{A} / \mathrm{m}=0.02 \mathrm{~m}^{2} / \mathrm{kg}$

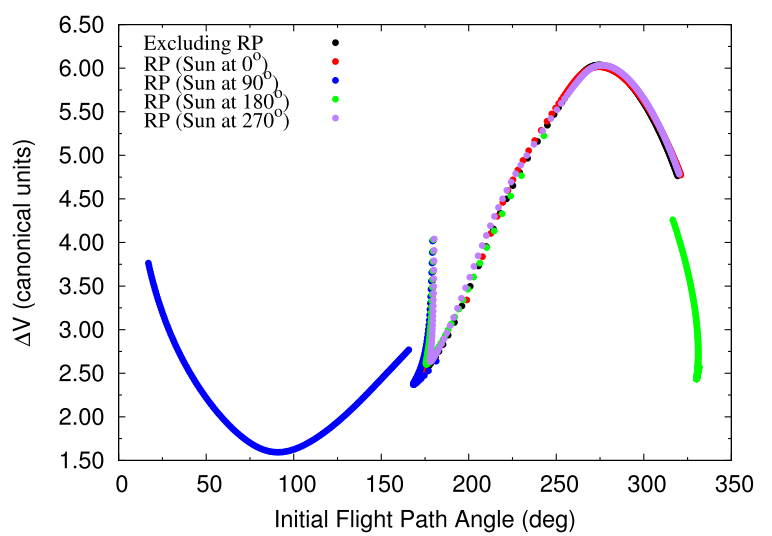

(d) $\Delta v$ as function of $f p a . \mathrm{A} / \mathrm{m}=0.1 \mathrm{~m}^{2} / \mathrm{kg}$

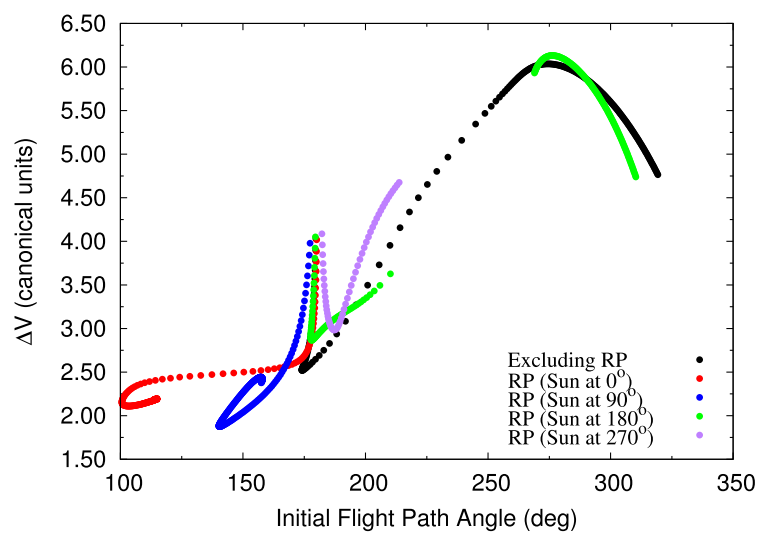

(f) $\Delta v$ as function of $f p a . \mathrm{A} / \mathrm{m}=0.5 \mathrm{~m}^{2} / \mathrm{kg}$

worst cases of 0.051 (about 2\%) for an area/mass ratio of $0.02 \mathrm{~m}^{2} ; 1.042$ (about $42 \%$ ) for an area/mass ratio of $0.1 \mathrm{~m}^{2}$ and 1.109 (about 44\%) for an area/mass ratio of $0.5 \mathrm{~m}^{2}$. Another important point shown in this table is related to the flight path angle at departure. They are similar for the area $/$ mass $=0.02 \mathrm{~m}^{2}$, but there are larger differences for the other higher values. For area/mass ratio $=0.1 \mathrm{~m}^{2}$, the values for the minimum increment of velocity are very different, 


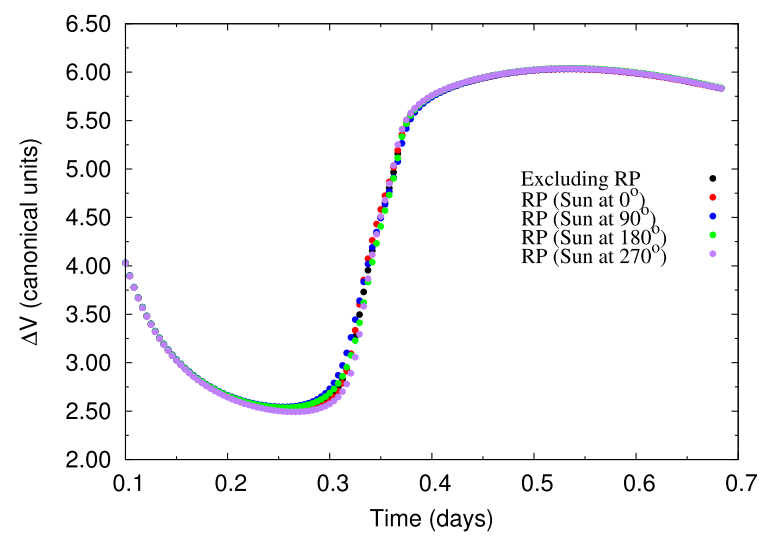

(a) $\Delta v$ as function of time. $\mathrm{A} / \mathrm{m}=0.02 \mathrm{~m}^{2} / \mathrm{kg}$

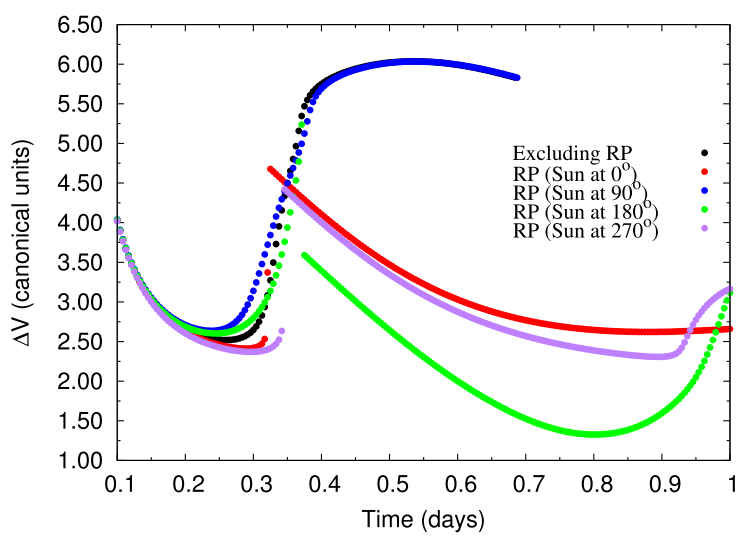

(c) $\Delta v$ as function of time. A/m $=0.1 \mathrm{~m}^{2} / \mathrm{kg}$

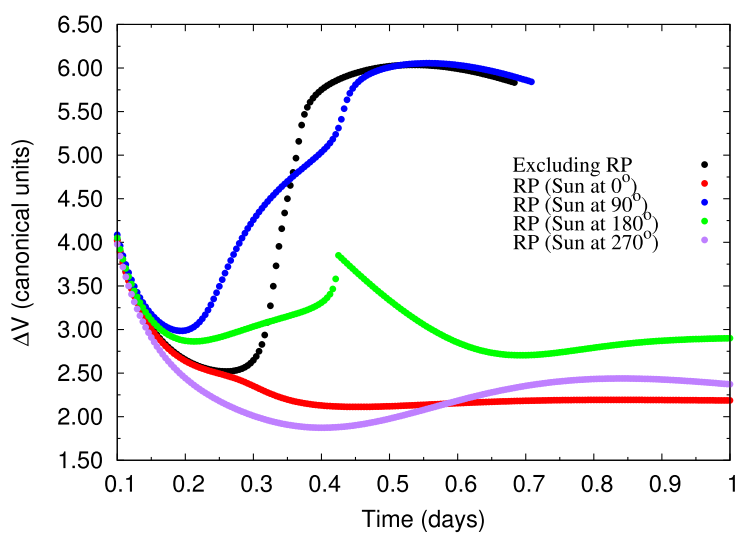

(e) $\Delta v$ as function of time. $\mathrm{A} / \mathrm{m}=0.5 \mathrm{~m}^{2} / \mathrm{kg}$

Fig. 9 Transfers from $L_{3}$ to $L_{1}$ with the asteroid at periapsis of its orbit

going from 90.894 degrees to 330.159 degrees, compared to the value of 174.113 degrees for the maneuver without solar radiation pressure. It means that, even in situations where the velocity increment is not much different, as in the case with the Sun initially at 180 degrees, with only 0.084 difference, the flight path angle moves from 174.113 degrees to 330.159 degrees. So, it is very important to take into account this force in the model. The same is true for the time of flight,

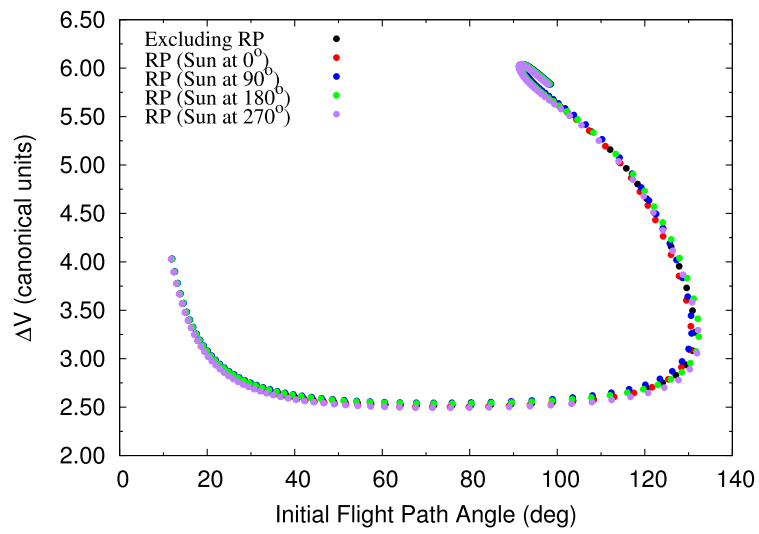

(b) $\Delta v$ as function of $f p a . \mathrm{A} / \mathrm{m}=0.02 \mathrm{~m}^{2} / \mathrm{kg}$

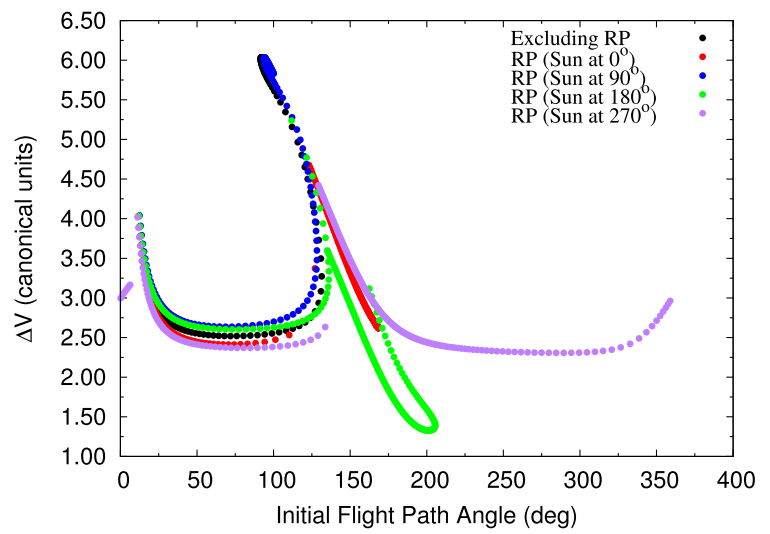

(d) $\Delta v$ as function of $f p a . \mathrm{A} / \mathrm{m}=0.1 \mathrm{~m}^{2} / \mathrm{kg}$

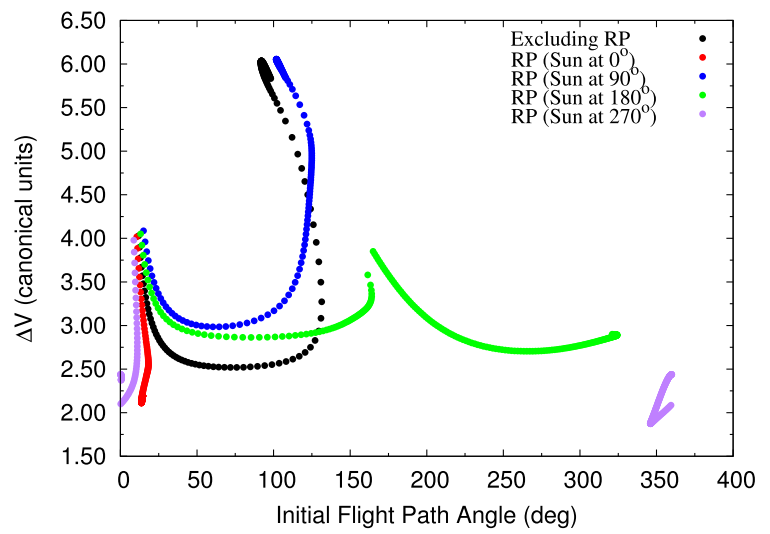

(f) $\Delta v$ as function of $f p a . \mathrm{A} / \mathrm{m}=0.5 \mathrm{~m}^{2} / \mathrm{kg}$

showing also larger variations for the minimum points. The reason for those large variations is the existence of several families of transfer orbits. They have similar values for all the three variables, velocity increment, flight path angle and transfer time. The border lines of those families have trajectories passing close to Alpha, and ending in collisions with the main asteroid. The presence of the solar radiation pressure makes shifts in those trajectories, changing the initial 
Table 4 1996FG 3 at periapsis, $\mathrm{L}_{1}$ to $\mathrm{L}_{3}$

\begin{tabular}{|c|c|c|c|c|c|c|c|c|c|}
\hline & \multicolumn{3}{|c|}{$A / m=0.02 \mathrm{~m}^{2} / \mathrm{kg}$} & \multicolumn{3}{|c|}{$A / m=0.1 \mathrm{~m}^{2} / \mathrm{kg}$} & \multicolumn{3}{|c|}{$A / m=0.5 \mathrm{~m}^{2} / \mathrm{kg}$} \\
\hline & $\Delta v_{\min }$ & fpa & time & $\Delta v_{\min }$ & fpa & time & $\Delta v_{\min }$ & fpa & time \\
\hline Excluding SRP & 2.519 & 174.113 & 0.258 & 2.519 & 174.113 & 0.258 & 2.519 & 174.113 & 0.258 \\
\hline Sun at $0^{\circ}$ & 2.500 & 173.570 & 0.263 & 2.410 & 169.386 & 0.287 & 2.110 & 103.483 & 0.454 \\
\hline Sun at $90^{\circ}$ & 2.493 & 173.229 & 0.267 & 1.593 & 90.894 & 0.692 & 1.874 & 140.527 & 0.399 \\
\hline Sun at $180^{\circ}$ & 2.537 & 174.555 & 0.254 & 2.435 & 330.159 & 0.858 & 2.866 & 178.082 & 0.212 \\
\hline Sun at $270^{\circ}$ & 2.544 & 175.032 & 0.254 & 2.635 & 178.107 & 0.237 & 2.983 & 187.447 & 0.196 \\
\hline
\end{tabular}

The units are: $\Delta v_{\min }$ (c.u.), fpa (degrees) and time (days)

Table $51996 \mathrm{FG}_{3}$ at periapsis, $\mathrm{L}_{3}$ to $\mathrm{L}_{1}$

\begin{tabular}{|c|c|c|c|c|c|c|c|c|c|}
\hline & \multicolumn{3}{|c|}{$A / m=0.02 \mathrm{~m}^{2} / \mathrm{kg}$} & \multicolumn{3}{|c|}{$A / m=0.1 \mathrm{~m}^{2} / \mathrm{kg}$} & \multicolumn{3}{|c|}{$A / m=0.5 \mathrm{~m}^{2} / \mathrm{kg}$} \\
\hline & $\Delta v_{\min }$ & fpa & time & $\Delta v_{\min }$ & fpa & time & $\Delta v_{\min }$ & fpa & time \\
\hline Excluding SRP & 2.519 & 71.815 & 0.258 & 2.519 & 71.815 & 0.258 & 2.519 & 71.815 & 0.258 \\
\hline Sun at $0^{\circ}$ & 2.500 & 71.407 & 0.263 & 2.412 & 70.472 & 0.288 & 2.112 & 13.746 & 0.454 \\
\hline Sun at $90^{\circ}$ & 2.544 & 71.751 & 0.254 & 2.636 & 68.668 & 0.238 & 2.985 & 60.363 & 0.192 \\
\hline Sun at $180^{\circ}$ & 2.537 & 71.806 & 0.254 & 1.326 & 200.982 & 0.800 & 2.704 & 265.280 & 0.692 \\
\hline Sun at $270^{\circ}$ & 2.493 & 75.602 & 0.267 & 2.307 & 289.410 & 0.896 & 1.873 & 346.036 & 0.400 \\
\hline
\end{tabular}

Table $61996 \mathrm{FG}_{3}$ at periapsis, $\mathrm{L}_{1}$ to $\mathrm{L}_{2}$

\begin{tabular}{|c|c|c|c|c|c|c|c|c|c|}
\hline & \multicolumn{3}{|c|}{$A / m=0.02 \mathrm{~m}^{2} / \mathrm{kg}$} & \multicolumn{3}{|c|}{$A / m=0.1 \mathrm{~m}^{2} / \mathrm{kg}$} & \multicolumn{3}{|c|}{$A / m=0.5 \mathrm{~m}^{2} / \mathrm{kg}$} \\
\hline & $\Delta v_{\min }$ & fpa & time & $\Delta v_{\min }$ & fpa & time & $\Delta v_{\min }$ & fpa & time \\
\hline Excluding SRP & 2.019 & 89.937 & 0.142 & 2.019 & 89.937 & 0.142 & 2.019 & 89.937 & 0.142 \\
\hline Sun at $0^{\circ}$ & 2.013 & 89.548 & 0.142 & 1.988 & 90.381 & 0.150 & 1.753 & 114.310 & 0.733 \\
\hline Sun at $90^{\circ}$ & 2.019 & 88.750 & 0.138 & 2.020 & 89.203 & 0.138 & 2.024 & 89.961 & 0.133 \\
\hline Sun at $180^{\circ}$ & 2.025 & 89.016 & 0.138 & 2.048 & 87.687 & 0.129 & 2.144 & 85.703 & 0.108 \\
\hline Sun at $270^{\circ}$ & 2.019 & 89.822 & 0.142 & 2.018 & 89.353 & 0.142 & 1.990 & 120.228 & 0.708 \\
\hline
\end{tabular}

conditions of the border lines. It means that some new families may appear due to those shifts, while some others may disappear due to collisions. This mechanism explains the appearance of new families with lower values of velocity increment. Figures 5 and 7 show this point, with trajectories that does not collide with Alpha when the solar radiation pressure is not considered, but that collides with Alpha after the inclusion of this force.

Figure 9 shows the results for transfers from $L_{3}$ to $L_{1}$ when the asteroid is in the periapsis of its orbit around the Sun. It is possible to observe, again, that the effects of the solar radiation pressure makes big changes over the transfers. As the area/mass ratio increases, more changes in $\Delta v$ can be observed. Depending on the initial position of the Sun, the values of $\Delta v$ decrease or increase. Table 5 shows a comparison of $\Delta v_{\min }, f p a$ and transfer time for all four cases of the initial position of the Sun and the situation with no solar radiation pressure. Table 5 has exactly the same physical in- terpretations of the large variations of the parameters of the minimum increment of velocities.

Tables 6 to 9 show a comparison of $\Delta v_{\text {min }}, f p a$ and transfer time for transfer involving all the others collinear Lagrange points transfers. A detailed analysis is not made because the phenomenon involved is very similar to the case $\mathrm{L}_{1}-\mathrm{L}_{3}$ shown in details before. This is the reason why only the tables are shown and the equivalent figures are omitted. Table 6 shows transfers from $L_{1}$ to $L_{2}$. Those two points have the body Beta in the middle, which is smaller than Alpha. It is the reason why there are fewer collisions with Beta, and there are not many alternations of transfer families. The most noted point occurs for an area/mass ratio of $0.5 \mathrm{~m}^{2} / \mathrm{kg}$ with the Sun located initially at 0 and $270 \mathrm{de}-$ grees. The case of 0 degree is particularly interesting, with larger savings in increment of velocity. This is due to the appearance of a family that ended in collisions when the solar radiation pressure is not considered. Table 7 shows the 
Table $7 \quad 1996 \mathrm{FG}_{3}$ at periapsis, $\mathrm{L}_{2}$ to $\mathrm{L}_{1}$

\begin{tabular}{|c|c|c|c|c|c|c|c|c|c|}
\hline & \multicolumn{3}{|c|}{$A / m=0.02 \mathrm{~m}^{2} / \mathrm{kg}$} & \multicolumn{3}{|c|}{$A / m=0.1 \mathrm{~m}^{2} / \mathrm{kg}$} & \multicolumn{3}{|c|}{$A / m=0.5 \mathrm{~m}^{2} / \mathrm{kg}$} \\
\hline & $\Delta v_{\min }$ & fpa & time & $\Delta v_{\min }$ & $f p a$ & time & $\Delta v_{\min }$ & fpa & time \\
\hline Excluding SRP & 2.204 & 155.651 & 0.167 & 2.204 & 155.651 & 0.167 & 2.204 & 155.651 & 0.167 \\
\hline Sun at $0^{\circ}$ & 2.204 & 155.238 & 0.167 & 2.204 & 155.238 & 0.167 & 2.217 & 123.026 & 0.288 \\
\hline Sun at $90^{\circ}$ & 2.205 & 155.693 & 0.163 & 2.205 & 155.693 & 0.163 & 2.245 & 148.599 & 0.142 \\
\hline Sun at $180^{\circ}$ & 2.110 & 326.143 & 1.000 & 2.110 & 326.143 & 1.000 & 2.215 & 165.261 & 0.142 \\
\hline Sun at $270^{\circ}$ & 2.202 & 156.375 & 0.167 & 2.202 & 156.375 & 0.167 & 2.089 & 278.733 & 0.183 \\
\hline
\end{tabular}

Table $81^{1996 F_{3}}$ at periapsis, $\mathrm{L}_{2}$ to $\mathrm{L}_{3}$

\begin{tabular}{|c|c|c|c|c|c|c|c|c|c|}
\hline & \multicolumn{3}{|c|}{$A / m=0.02 \mathrm{~m}^{2} / \mathrm{kg}$} & \multicolumn{3}{|c|}{$A / m=0.1 \mathrm{~m}^{2} / \mathrm{kg}$} & \multicolumn{3}{|c|}{$A / m=0.5 \mathrm{~m}^{2} / \mathrm{kg}$} \\
\hline & $\Delta v_{\min }$ & fpa & time & $\Delta v_{\text {min }}$ & $f p a$ & time & $\Delta v_{\min }$ & $f p a$ & time \\
\hline Excluding SRP & 2.193 & 319.008 & 0.912 & 2.193 & 319.008 & 0.912 & 2.193 & 319.008 & 0.912 \\
\hline Sun at $0^{\circ}$ & 2.176 & 317.989 & 0.983 & 2.274 & 320.212 & 0.829 & 2.442 & 113.372 & 0.771 \\
\hline Sun at $90^{\circ}$ & 2.172 & 320.174 & 0.883 & 2.117 & 322.706 & 0.812 & 2.024 & 321.448 & 0.654 \\
\hline Sun at $180^{\circ}$ & 2.215 & 319.110 & 0.879 & 2.310 & 316.636 & 0.800 & 2.672 & 302.516 & 0.675 \\
\hline Sun at $270^{\circ}$ & 2.220 & 317.265 & 0.950 & 2.366 & 315.560 & 0.862 & 2.890 & 316.339 & 0.742 \\
\hline
\end{tabular}

Table $91996 \mathrm{FG}_{3}$ at periapsis, $\mathrm{L}_{3}$ to $\mathrm{L}_{2}$

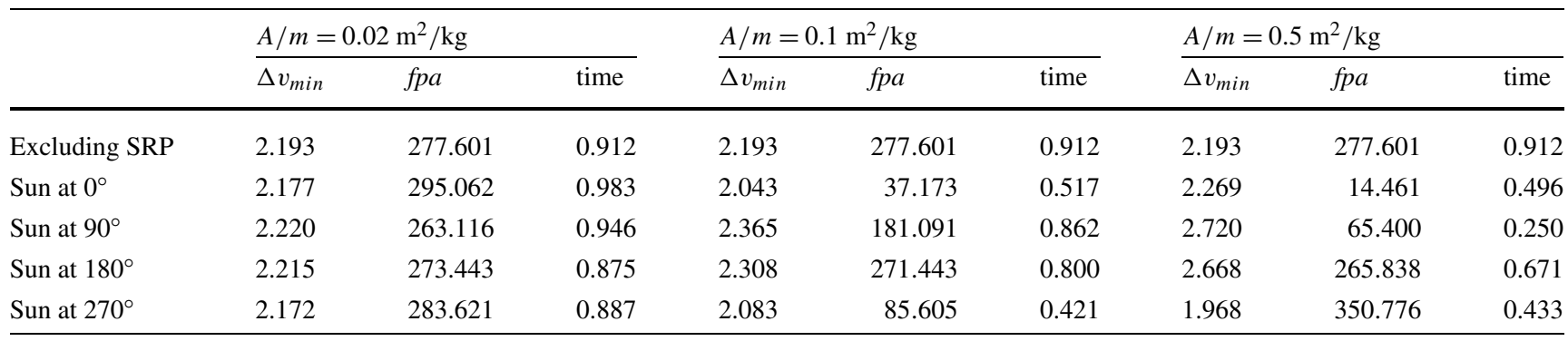

opposite transfer, from $\mathrm{L}_{2}$ to $\mathrm{L}_{1}$. The more interesting variation occurs when the Sun is located initially in 180 degrees, for the cases with area/mass of 0.02 and $0.1 \mathrm{~m}^{2} / \mathrm{kg}$. A new family with much longer transfer times and smaller velocity increments appear. Tables 8 and 9 consider transfers from $\mathrm{L}_{2}$ to $\mathrm{L}_{3}$ and vice-versa. Alpha is again in the middle of the transfers, so the alternations of families are back and families with lower increment of velocities appear again. The magnitudes of the modifications are smaller, because $L_{2}$ is far away from Alpha, compared to $\mathrm{L}_{1}$, and it reduces the frequency of collisions.

\section{Conclusion}

Transfers between the Lagrange points in a system of asteroids considering the effects of the solar radiation pressure were studied. It was found that the solar radiation pressure changes the trajectories performed by the spacecraft, modifying the energy required for the transfers.
The results show that the solar radiation pressure has strong effects in the dynamics. The differences in the variation of velocity between the many situations are larger and there are several cases where the solar radiation pressure decreases or increases the variation of velocity required for the transfer. The effects of the distance Sun-asteroid and the initial position of the Sun show the importance in considering the effects of the solar radiation pressure.

It is also an indication that the spacecraft would not reach the target, if the solar radiation pressure is not considered in the dynamics. This happens because, when considering smaller bodies like asteroids, the importance of the solar radiation pressure increases, and the locations and values of the best transfers are different. As shown in a previous work, Oliveira et al. (2016), the effects of the solar radiation pressure on transfers realized in the Sun-Earth system has a minimal effect. This indicates that, the force due to solar radiation pressure has a great effect in systems of small bodies, when compared to systems of larger bodies. 
Thus, it is possible to choose the right moment to perform the maneuver, such that the magnitudes of the impulses to be applied can be minimized. It is also shown an alternation of families to solve the transfer problem, because the solar radiation pressure influences the occurrence of collisions with the asteroids, which is the reason that ends the transfer orbit families. Therefore, the solar radiation pressure can be used also to reduce the magnitude of the increment of velocities required, reaching savings above $40 \%$ in some cases.

Acknowledgements The authors wish to express their appreciation for the support provided by grants 2011/13101-4, 2011/08171-3, 2014/22295-5 and 2016/14665-2, from São Paulo Research Foundation (FAPESP), grants 301338/2016-7, 4068411/2016-0 and 473164/ 2013-2 from the National Council for Scientific and Technological Development $(\mathrm{CNPq})$.

\section{References}

Broucke, R.: J. Guid. Control Dyn. 2(4), 257 (1979)

Cabette, R.E.S., Prado, A.F.: Acta Astronaut. 63(11), 1221 (2008)

Glassmeier, K.-H., Boehnhardt, H., Koschny, D., Kührt, E., Richter, I.: Space Sci. Rev. 128(1-4), 1 (2007)

Kawaguchi, J., Kuninaka, H., Fujiwara, A., Uesugi, T.: Acta Astronaut. 59(8), 669 (2006)

Lo, M., Williams, B., Bollman, W., Han, D., Hahn, Y., Bell, J., Hirst, E., Corwin, R., Hong, P., Howell, K.: In: AIAA/AAS Astrodynamics Specialist Conference and Exhibit, p. 4468 (1998)
Michel, P., Barucci, M.A., Cheng, A., Böhnhardt, H., Brucato, J.R., Dotto, E., Ehrenfreund, P., Franchi, I., Green, S., Lara, L.-M., et al.: Acta Astronaut. 93, 530 (2014)

Oliveira, G.M., Prado, A.F., Sanchez, D.M., Gomes, V.M.: In: 14th International Conference on Space Operations, p. 2558 (2016)

Prado, A.F.: Acta Astronaut. 39(7), 483 (1996)

Press, W.H., Flannery, B.P., Teukolsky, S.A., Vetterling, W.T., et al.: Numerical Recipes vol. 3, (1989)

Prockter, L., Murchie, S., Cheng, A., Krimigis, S., Farquhar, R., Santo, A., Trombka, J.: Acta Astronaut. 51(1), 491 (2002)

Rayman, M.D., Fraschetti, T.C., Raymond, C.A., Russell, C.T.: Acta Astronaut. 58(11), 605 (2006)

Scheirich, P., Pravec, P., Jacobson, S., Durech, J., Kušnirák, P., Hornoch, K., Mottola, S., Mommert, M., Hellmich, S., Pray, D., et al.: Icarus 245, 56 (2015)

Sukhanov, A., Velho, H.d.C., Macau, E., Winter, O.: Cosm. Res. 48(5), 443 (2010)

Szebehely, V.: Theory of Orbits, (1967)

Tsuda, Y., Mori, O., Funase, R., Sawada, H., Yamamoto, T., Saiki, T., Endo, T., Yonekura, K., Hoshino, H., Kawaguchi, J.: Acta Astronaut. 82(2), 183 (2013a)

Tsuda, Y., Yoshikawa, M., Abe, M., Minamino, H., Nakazawa, S.: Acta Astronaut. 91, 356 (2013b)

Vallado, D.A.: Fundamentals of Astrodynamics and Applications, vol. $12,(2001)$

Wolters, S.D., Rozitis, B., Duddy, S.R., Lowry, S.C., Green, S.F., Snodgrass, C., Hainaut, O.R., Weissman, P.: Mon. Not. R. Astron. Soc. 418(2), 1246 (2011)

Yang, H., Gong, S., Baoyin, H.: Astrophys. Space Sci. 357(1), 66 (2015) 